\title{
Patterns of local, intercontinental and interseasonal variation of soil bacterial and eukaryotic microbial communities
}

\author{
Johan De Gruyter ${ }^{1, *}$, , James T. Weedon ${ }^{1,2}$, Stéphane Bazot ${ }^{3}$, Steven Dauwe ${ }^{1}$, \\ Pere-Roc Fernandez-Garberí ${ }^{4,5}$, Stefan Geisen ${ }^{6}$, Louis Gourlez De La Motte ${ }^{7}$, \\ Bernard Heinesch ${ }^{7}$, Ivan A. Janssens ${ }^{1}$, Niki Leblans ${ }^{1}$, Tanguy Manise ${ }^{8}$, \\ Romà Ogaya ${ }^{4,5}$, Mikaell Ottosson Löfvenius ${ }^{9}$, Josep Peñuelas ${ }^{4,5}$, Bjarni \\ D. Sigurdsson ${ }^{10}$, Gaëlle Vincent ${ }^{3}$ and Erik Verbruggen ${ }^{1}$
}

${ }^{1}$ Centre of Excellence PLECO (Plants and Ecosystems), Department of Biology, University of Antwerp, 2610 Wilrijk, Belgium, ${ }^{2}$ Department of Ecological Sciences, Vrije Universiteit Amsterdam, 1081 HV Amsterdam, the Netherlands, ${ }^{3}$ Ecologie Systématique Evolution, University of Paris-Sud, CNRS, AgroParisTech, Université Paris-Saclay, 91400 Orsay, France, ${ }^{4}$ CREAF, Cerdanyola del Vallès, Barcelona 08193, Catalonia, Spain, ${ }^{5}$ CSIC, Global Ecology Unit CREAF-CSIC-UAB, Bellaterra, Barcelona 08193, Catalonia, Spain, ${ }^{6}$ Department of Terrestrial Ecology, Netherlands Institute of Ecology (NIOO-KNAW), 6708 PB Wageningen, the Netherlands, ${ }^{7}$ TERRA Teaching and Research Centre, Gembloux Agro-Bio Tech, University of Liege, B-5030 Gembloux, Belgium, ${ }^{8}$ AGROBIOCHEM Research Unit, Gembloux Agro-Bio Tech, University of Liege, B-5030 Gembloux, Belgium, ${ }^{9}$ Department of Forest Ecology and Management, Swedish University of Agricultural Sciences (SLU), SE-901 83 Umea, Sweden and ${ }^{10}$ Faculty of Environmental Sciences, Agricultural University of Iceland, Hvanneyri, IS-311 Borgarnes, Iceland

*Corresponding author:Centre of Excellence PLECO (Plants and Ecosystems), Department of Biology, University of Antwerp, 2610 Wilrijk, Belgium. Tel: 003232651715; E-mail: johan.degruyter@uantwerpen.be

One sentence summary: Soil microbes exhibit strong spatial patterns determined by their size as well as co-changing temporal patterns between groups.

Editor: Ian Anderson

†Johan De Gruyter, http://orcid.org/0000-0001-5020-8633

\section{ABSTRACT}

Although ongoing research has revealed some of the main drivers behind global spatial patterns of microbial communities, spatio-temporal dynamics of these communities still remain largely unexplored. Here, we investigate spatio-temporal variability of both bacterial and eukaryotic soil microbial communities at local and intercontinental scales. We compare how temporal variation in community composition scales with spatial variation in community composition, and explore the extent to which bacteria, protists, fungi and metazoa have similar patterns of temporal community dynamics. All soil 
microbial groups displayed a strong correlation between spatial distance and community dissimilarity, which was related to the ratio of organism to sample size. Temporal changes were variable, ranging from equal to local between-sample variation, to as large as that between communities several thousand kilometers apart. Moreover, significant correlations were found between bacterial and protist communities, as well as between protist and fungal communities, indicating that these microbial groups change in tandem, potentially driven by interactions between them. We conclude that temporal variation can be considerable in soil microbial communities, and that future studies need to consider temporal variation in order to reliably capture all drivers of soil microbiome changes.

Keywords: soil microbial communities; spatio-temporal variability; microbial ecology; protists; soil biogeography

\section{INTRODUCTION}

In recent years, the development of high-throughput sequencing (HTS) techniques has allowed for rapid developments in the field of molecular ecology, particularly for characterization of microbial communities. The main advantages of HTS techniques are that they are culture independent and that they generate tremendous amounts of sequence data at a fraction of the cost of earlier methods (Caron et al. 2011; Logares et al. 2012; Lie et al. 2014; Mahé et al. 2015). Thanks to this increased capacity to census microbial communities through environmental barcoding, global efforts are being made to map the soil microbiome and predict corresponding community structures based on abiotic and biotic parameters.

These studies have to date mainly focused on bacteria and fungi. Consequently, we now know that $\mathrm{pH}$ is often found to be a major driver of changes in bacterial and archaeal community composition on both the local scale and the global scale (Lauber et al. 2009; Bahram et al. 2018; Delgado-Baquerizo et al. 2018a; Delgado-Baquerizo et al. 2018b). However, other studies have found that various other factors can also be important in explaining global patterns for soil bacterial communities. For example, both Maestre et al. (2015) and Zhou et al. (2016) have shown that climatic factors such as aridity and temperature can shape soil bacterial communities. Similarly, vegetation type (Prober et al. 2015) and nutrient availability (Leff et al. 2015) have also been shown to have a significant influence. Most studies agree that a low number of bacterial species are usually shared between sites, but also that a small amount of species usually makes up nearly half of the recovered sequences and that these dominant species can often be the ones shared between distinct sites (Ramirez et al. 2014; Maestre et al. 2015; DelgadoBaquerizo et al. 2018a). And although these dominant species are usually spread out over several phylogenetic groups, more than half of them can be divided into groups with predictable habitat preferences (Delgado-Baquerizo et al. 2018a). Interestingly, several studies seem to find that spatial distance is only a weak predictor for community dissimilarity for bacteria, suggesting that environmental parameters are more important in shaping bacterial communities than dispersal capacity (Lauber et al. 2009; Bahram et al. 2018). This is in contrast to fungal communities, where geographic distance has been found to be an important predictor of community composition (Bahram et al. 2018). This illustrates that fungi and bacteria have different drivers for community assembly on the local and the global scale (Rousk et al. 2010; Bahram et al. 2018). Indeed, other studies regarding fungal composition on a global scale find that dispersal is a likely candidate for driving global fungal patterns. For example, Treseder et al. (2014) found that the youngest clades of fungi appear closer to the poles, suggesting a dispersion starting from the tropical regions outward. A similar result was found by Talbot et al. (2014), who found strong geographical cluster- ing of fungal communities, suggesting a dispersal limit. In contrast to these studies highlighting biogeographic patterns, variation in other edaphic parameters such as vegetation and climatic factors has been proposed to be as important, or perhaps even more important in shaping soil fungal communities on the global scale (Tedersoo et al. 2014, 2016). In addition to these environmental filters, there is of course an important role for biological interactions between organisms in shaping community composition. Bahram et al. (2018), for example, found evidence for the influence of biotic interactions on both bacterial and fungal communities. However, soils are among the most complex habitats on Earth and consequently host an enormous diversity of functionally different microbes besides bacteria and fungi. How assemblages of these other organisms vary with their environment and with other microbes is much less studied. For example, protists are by far the most diverse and (together with fungi) the most abundant soil eukaryotes (Ekelund and Rønn 1994; Foissner 1997; Finlay et al. 2000). Different taxa of soil protists are known to function as consumers of bacteria, fungi and other protists (Clarholm 1981; de Ruiter, Neutel and Moore 1995; Geisen et al. 2015b; Mitchell 2015), consume metazoans or act as parasites (Geisen et al. 2015a, 2016; Mitchell 2015). Protists are therefore a crucial component of the soil microbial community, yet their response to environmental changes or community shifts of other microbial groups remains largely unknown (Geisen et al. 2017).

While spatial patterns of major microbial groups are increasingly being mapped, temporal dynamics have received far less attention in soils. This is surprising, given that some earlier studies on soil bacteria have clearly illustrated that communities do exhibit interseasonal changes, which can often be linked to temporal differences in environmental parameters. For example, Lauber et al. (2013) found that soil bacterial communities from bulk soil exhibited temporal patterns that were significantly determined by changing soil moisture and temperature. Similarly, Lazzaro, Hilfiker and Zeyer (2015) and Zhang et al. (2018) found that seasonal changes in temperature, $\mathrm{pH}$ and nutrients had a large influence on soil bacterial communities in alpine sites, while Chemidlin Prevost-Boure et al. (2011) found both soil moisture and litter amount to be determining factors for seasonal changes in soil bacteria in a temperate deciduous forest. Apart from these abiotic parameters, the plant community can also exert an influence on soil microbial communities that changes over time (Yao, Bowman and Shi 2011). From these results, it becomes clear that the factors influencing soil microbial communities on the temporal scale are very similar to the factors influencing community composition on the spatial scale. Considering these factors can exhibit equally large changes over seasons as they can when comparing spatially distant sites, they are expected to correlate with similar degrees of variation in soil microbial communities. Moreover, this additional source of variation may potentially confound interpretations of spatial 
patterns in communities. To date, however, the combination of spatial and temporal variability of soil microbial communities, especially eukaryotic soil microbial communities, has not yet been systematically examined. As any study on temporal variation will invariably contain some spatial variation on a local scale (as repeated soil sampling in the exact same location is impractical because of disturbance), it is important to know the relative size of both to avoid confounding spatial and temporal patterns when interpreting data from environmental sampling.

This study aims to investigate spatio-temporal variation of both bacterial and eukaryotic soil microbial communities using a multiscale approach including local and intercontinental variation within and between sampling occasions at different times of the year. We characterize temporal changes in soil microbial communities to investigate the relative size of temporal and spatial variation, and whether the degree of spatio-temporal variation differs between bacteria, fungi, protists and metazoa. Additionally, the correlation between site characteristics and the degree of interseasonal variation is tested. This knowledge is important as it will allow us to estimate the prevalence and relative size of spatial and interseasonal variation in soil microbial communities.

\section{METHODOLOGY}

\section{Study sites and sampling}

The sites selected for this study are part of ongoing experiments and studies (Table S1, Supporting Information) and thus provide an opportunity to study soils in well-characterized ecological settings. A total of 13 sites were sampled from seven countries (Australia, Belgium, France, French Guiana, Iceland, Spain and Sweden) spanning five climate types (temperate oceanic, subarctic, tropical rainforest, humid subtropical and Mediterranean) and eight different ecosystem types (broadleaf forest, cereal cropland, coniferous forest, eucalypt forest, grassland, holm oak forest, shrubland and tropical forest). This information is listed in Table 1, and additional information regarding specific site characteristics are provided in Table S2 (Supporting Information). Each site was sampled at two distinct time points, which were selected based on expected differences in microbial activity. At every sampling, four replicates were taken from the site (except in the Garraf, Prades, Reykir and French Guiana sites, where eight, nine, five and five samples were taken, respectively), for a total of 154 samples.

For each site, we obtained soil $\mathrm{pH}$, average vegetation height and elevation above sea level (Table S2, Supporting Information). As climate variables, the average minimum and maximum temperature and total precipitation for the month leading up to the sample date were obtained from on-site weather stations, or when this was not available, from the nearest weather station (Reykir, Nouragues). Soil samples were taken at random locations within an $\sim 20 \mathrm{~m} \times 20 \mathrm{~m}$ area using a corer. The upper 5 to $10 \mathrm{~cm}$ soil layer was sampled directly below the bottom of the 'intact litter' layer. If no litter was present, the upper 0$15 \mathrm{~cm}$ of the soil was sampled. After sampling, soil was frozen and stored at $-20^{\circ} \mathrm{C}$ until further processing, with the exception of the two tropical sites, where samples were frozen and subsequently freeze-dried before storage at room temperature. The deviating storage procedure in the tropical sites was due to the unavailability of reliable freezers.

\section{Library preparation and sequencing}

DNA was extracted from $\sim 0.25 \mathrm{~g}$ of soil using the MoBio Powersoil kit according to the manufacturer's protocol (MoBio,

Table 1. Comprehensive list of all sampling sites, including information about climate (based on Köppen-Trewartha classification), location, country, sampling time and ecosystem type.

\begin{tabular}{|c|c|c|c|c|}
\hline Location & Country & Climate & Ecosystem type & Sampling time \\
\hline Brasschaat & Belgium & Temperate oceanic & Coniferous forest & $\begin{array}{l}\text { End of spring (June, high expected } \\
\text { microbial activity) and end of autumn } \\
\text { (November, low expected microbial activity) }\end{array}$ \\
\hline Dorinne & & & Grassland & \\
\hline Lonzee & & & Cereal cropland & \\
\hline Barbeau & France & & Oak forest & \\
\hline Reykir & Iceland & Subarctic & Grassland & $\begin{array}{l}\text { Spring (April, low expected microbial } \\
\text { activity) and autumn (October, high } \\
\text { expected microbial activity) }\end{array}$ \\
\hline Svartberget & Sweden & & Coniferous forest & $\begin{array}{l}\text { Spring (June, high expected microbial } \\
\text { activity) and autumn (November, low } \\
\text { expected microbial activity) }\end{array}$ \\
\hline \multirow[t]{2}{*}{ Nouragues } & French Guiana & Tropical rainforest & Tropical forest hilltop & $\begin{array}{l}\text { Wet season (June, high expected microbial } \\
\text { activity) and dry season (October, low } \\
\text { expected microbial activity) }\end{array}$ \\
\hline & & & Topical forest valley & \\
\hline Paracou & & & $\begin{array}{l}\text { Tropical forest hilltop } \\
\text { Tropical forest valley }\end{array}$ & \\
\hline Cumberland & Australia & Humid subtropical & Eucalypt forest & $\begin{array}{l}\text { Winter (June, low expected microbial } \\
\text { activity) and summer (December, high } \\
\text { expected microbial activity) }\end{array}$ \\
\hline Garraf & Spain & $\begin{array}{l}\text { Mediterranean hot } \\
\text { summer }\end{array}$ & Shrubland & $\begin{array}{l}\text { Spring (April, low expected microbial } \\
\text { activity) and summer (August, high } \\
\text { expected microbial activity) }\end{array}$ \\
\hline Prades & & & Holm oak forest & \\
\hline
\end{tabular}


Carlsbad, CA, USA), and all extracts were measured using a Qubit 3.0 Fluorometer (Invitrogen, Waltham, MA, USA) and standardized to $5 \mathrm{ng} / \mu \mathrm{l}$ to reduce variability (Kennedy et al. 2014). For eukaryotes, we used the general TarEuk primers described by Stoeck et al. (2010): TAReuk454FWD1 [5'-CCAGCA(G/ C)C(C / T)GCGGTAATTCC-3', S. cerevisiae position 565-584] and TAReukREV3 [5'-ACTTTCGTTCTTGAT(C / T) (A / G)A-3', S. cerevisiae position 964-981], targeting the hypervariable V4 region of the 18S rRNA gene. These primers were selected not only as they have been used previously in studies looking at protist communities (Mahé et al. 2017) but also for the examination of fungal and metazoan community composition (Bernhard et al. 2014; Pawlowski et al. 2014; Dunthorn et al. 2017; López-Escardó et al. 2018). An adapter was included in the primers for attaching indices after the initial polymerase chain reaction (PCR). We also used a newly designed plant blocker (PNA 5'-GCTCAAAGCAAGC3') to limit the amplification of plant DNA in the samples. The blocker was designed by aligning the sequence with a wide range of plant species and representatives from all major supergroups of protists, fungi and metazoa to ensure the blocker selects against most embryophyte species, while allowing amplification of single-celled chlorophytes. We optimized the reaction mix by varying annealing temperature, primer and blocker concentrations and visually inspecting amplicon size and intensity on a $1.5 \%$ agarose gel. The optimized $25 \mu \mathrm{l}$ reaction mixture contained $1.5 \mu \mathrm{l}$ of sample, $0.5 \mu \mathrm{M}$ of each primer, $0.8 \mu \mathrm{M}$ plant blocker and $1 \times$ Phusion High-Fidelity PCR Master Mix with GC Buffer (ThermoFisher Scientific, Waltham, MA, USA). The PCR profile was as follows: an initial denaturation step at $98^{\circ} \mathrm{C}$ for 5 min, followed by 35 cycles of $98^{\circ} \mathrm{C}$ for $45 \mathrm{~s}$, annealing of the plant blocker at $67^{\circ} \mathrm{C}$ for $20 \mathrm{~s}$, primer annealing at $54^{\circ} \mathrm{C}$ for $60 \mathrm{~s}$ and elongation at $72^{\circ} \mathrm{C}$ for $90 \mathrm{~s}$, with a final elongation step at $72^{\circ} \mathrm{C}$ for $10 \mathrm{~min}$. Amplified products were purified using SequalPrep according to the manufacturer's protocol (Thermo Fisher Scientific, Waltham, MA, USA), after which a second PCR was performed to attach the Illumina indices to the product (Illumina, San Diego, CA, USA). This PCR had the following reaction mix: $2.5 \mu$ l of purified PCR amplicon, $0.2 \mu \mathrm{M} \mathrm{p5/p7} \mathrm{primer} \mathrm{mix,}$ $1 \times$ Phusion High-Fidelity PCR Master Mix with GC Buffer and the following PCR profile: $98^{\circ} \mathrm{C}$ for $60 \mathrm{~s}$, followed by 10 cycles of $98^{\circ} \mathrm{C}$ for $10 \mathrm{~s}$, primer annealing at $55^{\circ} \mathrm{C}$ for $30 \mathrm{~s}$, elongation at $72^{\circ} \mathrm{C}$ for $30 \mathrm{~s}$ and final elongation at $72^{\circ} \mathrm{C}$ for $5 \mathrm{~min}$. A second purification and equalization was done with SequalPrep with a slight modification of the protocol to increase the final eluate concentration. This was followed by agarose gel electrophoresis and extraction to exclude primer-dimers, after which the concentration of the pooled finished library was determined by qPCR and sequenced on an Illumina Miseq using $2 \times 300$ cycles of pairedend sequencing.

For bacteria, we used the primers targeting the hypervariable V3-V4 region of the 16S rRNA gene (S-D-Bact-0341-b-S17, 5'-CCTACGGGNGGCWGCAG-3' and S-D-Bact-0785-a-A-21, 5'-GACTACHVGGGTATCTAATCC -3') as described by Klindworth et al. (2013). The reaction mixture contained $1.5 \mu \mathrm{l}$ of sample, $0.5 \mu \mathrm{M}$ of each primer and $1 \times$ Phusion Master Mix. The PCR conditions were $98^{\circ} \mathrm{C}$ for $5 \mathrm{~min}, 25$ cycles of $98^{\circ} \mathrm{C}$ for $40 \mathrm{~s}, 55^{\circ} \mathrm{C}$ for $30 \mathrm{~s}, 72^{\circ} \mathrm{C}$ for $60 \mathrm{~s}$ and a final extension at $72^{\circ} \mathrm{C}$ for $10 \mathrm{~min}$. After amplification, the subsequent steps were the same as for eukaryotes. To test for the preservation of community fidelity, chimera formation and primer bias, several internal controls from representatives of most of the major important groups found in soils were selected. These internal controls were spiked into extracts of four samples from different ecosystems to cover as broad a spatial and season gradient as possible, but without performing the analysis on all samples. The amount of internal controls that was added to each sample was such that the controls should represent at least $30 \%$ of the total 18S rRNA gene concentration (see the Supporting Information).

\section{Bioinformatics and statistical analysis}

Eukaryotic paired-end sequences were merged using the VSEARCH V2.5.I 'merge' function (Rognes et al. 2016), successfully merging $51.4 \%$ of the sequences; this relative low percentage of successfully merged sequences was caused by increasing error rates toward the end of the run. Bacterial sequences were not merged because $3^{\prime}$ quality dropped sharply, so that merging would have resulted in retaining few sequences. Instead, only the forward sequences were used after trimming to a length of $200 \mathrm{bp}$. Primer sequences were removed from all reads, and reads were subsequently quality-filtered allowing for a maximum expected error of 0.6 , leaving 2.7 and $3.1 \mathrm{M}$ reads for eukaryotes and bacteria, respectively. Operational taxonomic units (OTUs) with $97 \%$ similarity were clustered de novo using the UPARSE algorithm (Edgar 2013) in USEARCHIO (Edgar 2010) after dereplication and singleton removal. After chimera removal (leaving 13387 and 14219 non-chimeric OTUs for eukaryotes and bacteria, respectively), all original reads were mapped to the non-chimeric OTUs (matching 1.9 and $2.7 \mathrm{M}$ reads for eukaryotes and bacteria, respectively) using USEARCHIO with the identity threshold of 0.97 to create an OTU table.

For the taxonomic assignment of OTUs, all eukaryotic OTUs were first aligned to the Protist Ribosomal Reference database version 4.6 (PR2; Guillou et al. 2013) using the default UCLUST function in QIIME (Caporaso et al. 2010; Edgar 2010) with an $80 \%$ sequence similarity threshold to compensate for potential higher divergence from known sequences. This threshold was selected because eukaryotic assignments with $<80 \%$ similarity to known references are often considered spurious (Stoeck et al. 2010; Mahé et al. 2017). Non-protist OTUs were subsequently aligned to the SILVA rRNA database version 128 (Quast et al. 2013), again with an $80 \%$ similarity threshold, after which the results from both databases were merged. This was done to increase non-protist coverage, as the PR2 database contains less non-protist sequences (Guillou et al. 2013). Eukaryotic OTUs were classified as protists, fungi or metazoa based on their best hit against the databases. Bacterial sequences were matched to the SILVA rRNA database using the UCLUST function in QIIME with a $90 \%$ similarity threshold as is the default setting. Plant sequences were also removed from the dataset as they were not the subject of study. To avoid library size-related artifacts in downstream data analysis, rarefied OTU tables were created by random subsampling of the original OTU tables split by taxonomic group (protists, fungi, metazoa and bacteria). We chose to apply a two-boundary approach (i.e. using separate values for the maximum read number and the minimum read number) for subsampling instead of a single-boundary subsampling (i.e. using a single value for minimum and maximum read number) to avoid missing temporal effects on microbial communities. This could have occurred because some time/group combinations had relatively few reads. Given that the eukaryotic community is sequenced as a whole, separate groups may have shifting read abundances over time, and thus a stringent subsampling threshold might eliminate all samples from a time point due to a shift in the total eukaryotic microbial community. Using both a minimum read number to avoid including failed samples and a maximum read number to avoid large size-related artifacts, and to normalize read numbers, any possible temporal abundance 
effect is preserved. Only six, four, four and five samples yielded read numbers below the lower thresholds for bacteria, protists, fungi and metazoa, respectively, and were considered as failed. We show that this approach does not greatly affect Bray-Curtis dissimilarities when OTU tables are normalized by scaling read numbers to 1 for each sample (Figures S4-S7, Supporting Information). All analyses were performed in $\mathrm{R}$ version 3.5.3 unless stated otherwise (R Core Team 2019).

Non-metric multidimensional scaling was performed to visualize the overall differences in microbial community composition for each group (Figures S8-S11, Supporting Information) and community Bray-Curtis dissimilarities between sites and time points were calculated using the VEGAN package version 2.5-5 (Oksanen et al. 2019) and the mean dissimilarities were tested for significant differences using analysis of variance (ANOVA). To assess intra-annual changes in community composition, two values were calculated, which we here refer to as 'turnover' and 'difference in variability'. Turnover was defined as the distance between Bray-Curtis centroids of both time points. The difference in variability was measured by first calculating the Bray-Curtis distance of each sample to its corresponding seasonal centroid, and then computing the absolute difference of the time point means of these values. Therefore, this value is small when the degree of within-time point variation between replicates is similar for each time point, and large when this is not the case. All these values were calculated using the USEDIST package version 0.1.0 in R (Bittinger 2017). We tested whether there was a relationship of turnover and difference in variability with edaphic parameters (temperature, precipitation, $\mathrm{pH}$ and elevation) using linear regression. Pearson correlation coefficients for turnover and difference in variability were computed for all pairwise combinations of the taxonomic groups. Spatial distance between sites was calculated based on coordinates using the GEOSPHERE package version 1.5-7 (Hijmans 2017). A Mantel test with 1000 permutations was used to estimate the significance of the relation between community dissimilarity and spatial distance. Furthermore, a linear regression model was fitted, plotting community dissimilarity, computed for all pairwise combinations excluding intra-annual community dissimilarity, against their corresponding spatial distance to estimate the magnitude of spatial and intra-annual dissimilarity. The resulting model fit was used to calculate the predicted spatial distance corresponding to each intra-annual dissimilarity observed. To estimate the significance of observed differences between groups in the intercept and slope of the linear models, the $95 \%$ quantile, median and mean were calculated for each group. To achieve this, we randomly subsampled the available sample-sample pairs in such a way that each specific site only occurs once in the subsampled dataset. This subset was then used to calculate the intercept and slope and the process was repeated 1000 times to generate the $95 \%$ quantile.

\section{RESULTS}

\section{Temporal and spatial effects on microbial groups}

Community dissimilarity did not show a consistent pattern when comparing samples from a single time point and samples between time points for the different taxonomic groups. Community dissimilarities within a given time point also varied greatly (Fig. 1), causing mean community dissimilarity between time points to be rarely higher than within each time point ( $P$ values for all comparisons $>0.05$ ). Within each microbial group, mean community dissimilarity between sites was consistently higher than both mean community dissimilarities within and between time points $(P$-values for all groups $<0.001)$. On average, bacteria showed the lowest mean community dissimilarities and metazoa the highest, with fungi and protists intermediate and comparable ( $P$-values for all comparisons $<0.001)$.

In agreement with the results for community dissimilarity, average turnover (the distance between temporal centroids) was also lowest for bacteria and highest for metazoa (bacteria: mean $=0.199$, standard error $(\mathrm{SE})=0.019$; fungi: mean $=0.320, \mathrm{SE}$ $=0.025$; protists: mean $=0.318, \mathrm{SE}=0.022$; metazoa: mean $=$ $0.401, \mathrm{SE}=0.020$ ). Therefore, the composition of bacterial communities appears to be more 'stable'. This is also reflected in a higher percentage of shared taxa between time points (Table 2). In contrast, metazoan communities have the lowest percentage of shared taxa between time points (bacteria: mean $=52.9 \%$, SE $=1.7$; fungi: mean $=45.2 \%, \mathrm{SE}=1.9$; protists: mean $=43.5 \%$, $\mathrm{SE}$ $=2.7$; metazoa: mean $=36.1 \%, \mathrm{SE}=3.3$ of reads shared). Similar to turnover, difference in variability (the difference between time points in how spread out the replicates are) was lowest for bacteria and highest for metazoa (bacteria: mean $=0.071, \mathrm{SE}=$ 0.015 ; fungi: mean $=0.125, \mathrm{SE}=0.033$; protists: mean $=0.103, \mathrm{SE}$ $=0.027$; metazoa: mean $=0.137, \mathrm{SE}=0.024)$. We did not find any significant relation between interseasonal changes in environmental parameters and interseasonal changes in communities (either turnover- or difference in variability-wise).

We found a significant correlation between bacterial turnover (the distance between temporal centroids) and protist turnover $(R=0.79, P=0.001)$. There was also a non-significant trend in the correlation between protist turnover and fungal turnover $(R=0.52, P=0.07)$. These correlations show that if the community dissimilarity between our interseasonal sampling occasions is large at a given site for protists, then the dissimilarity between sampling occasions of bacteria (and possibly fungi) is also high at the same site. Similarly, the difference in variability of bacteria (the difference in spread of the communities around their temporal centroid) correlates with that of all other groups (bacteria-fungi: $R=0.66, P=0.01$; bacteria-metazoa: $R=0.57, P=0.04$; bacteria-protists: $R=$ $0.91, P<0.0001$ ), and protist difference in variability correlates significantly with fungal difference in variability $(R=0.86$, $P=0.0002$ ). A positive correlation of difference in variability between two groups indicates that when samples differ more in one time point than another for one group, the same is true for the other group. These findings are shown in Fig. 2.

Community dissimilarity significantly increases as the spatial distance between sites increases for all groups (bacteria: Mantel statistic $R=0.67$, permutation $P=0.001$; protist: $R=0.79$, $P=0.001$; fungi: $R=0.74, P=0.001$; metazoa: $R=0.51, P=0.001$ ). The slope shows a decreasing trend in the order (bacteria: slope $=0.094$, intercept $=0.17$; protists: slope $=0.061$, intercept $=0.52$; fungi: slope $=0.059$, intercept $=0.51$; metazoa: slope $=0.032$, intercept $=0.72$ ), while the intercept shows the opposite trend (Fig. 3). With the exception of the comparison between fungi and protists, these values fall outside of the calculated $95 \%$ quantiles (Tables S3 and S4, Supporting Information). It is of importance to note that many of the spatially distant sites have different ecosystems; therefore, the larger community dissimilarity at these spatial scales could also be due to different environments and not strictly spatial. Interseasonal community dissimilarity varied greatly, with the majority of values equivalent to dissimilarities corresponding to spatial distances of between 1 and $100 \mathrm{~m}$, i.e. falling within the range of local spatial variation. However, some of the extremes displayed dissimilarities that would correspond with spatial distances of $>1000 \mathrm{~km}$ (Fig. 3). 


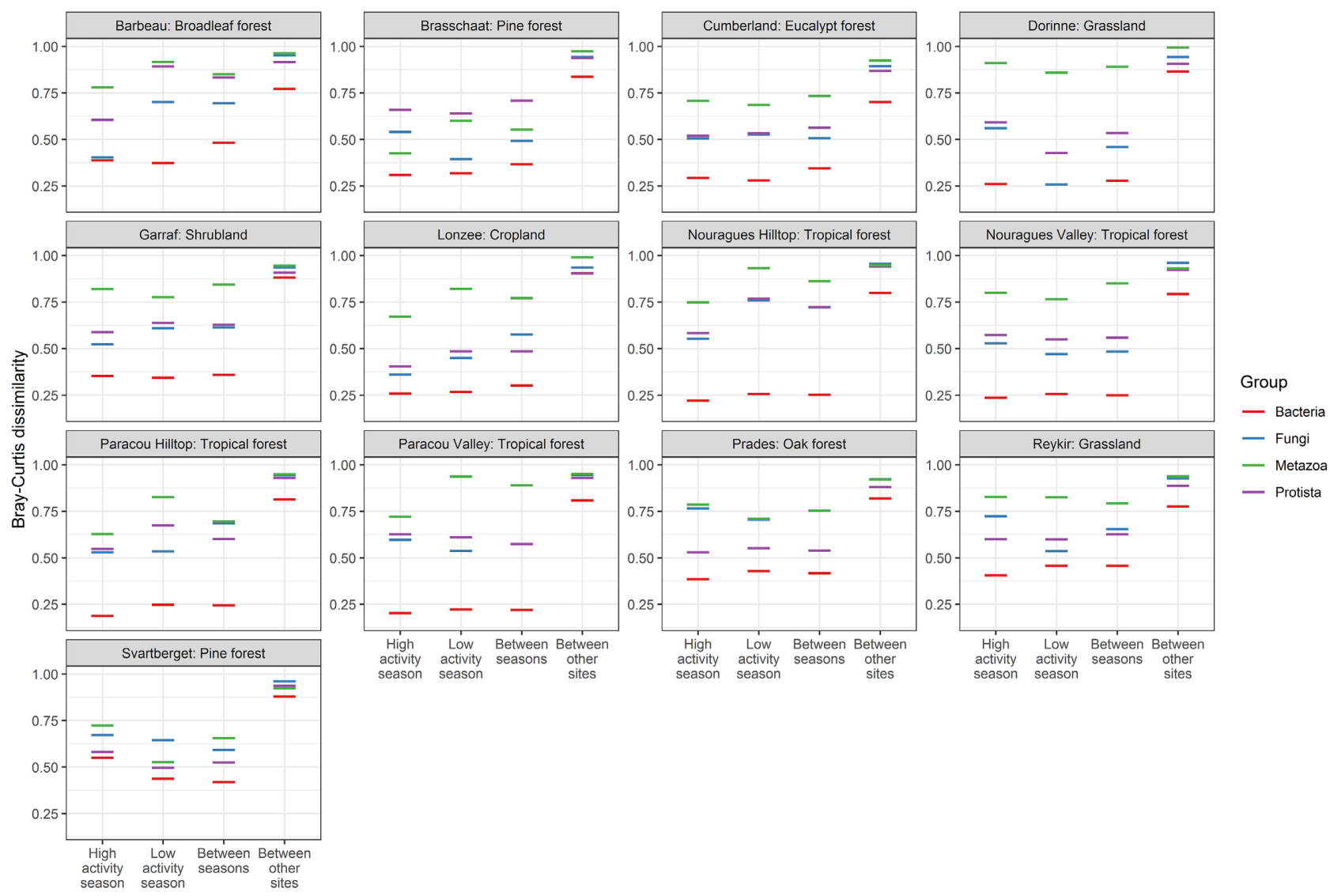

Figure 1. Within-site comparisons of median community dissimilarities within ('High activity season', 'Low activity season') and between ('Between seasons') time points, as well as with other sites ('Between other sites') for each of the different taxonomic groups. 'High activity season' represents the time point where the highest microbial activity is expected, and 'Low activity season' represents the time point with the lowest expected microbial activity. Bacteria are colored in red, fungi in blue, metazoa in green and protists in purple. Error bars are omitted for clarity.

The results of the methodological controls illustrate that resident community rank abundance is not altered when introducing large quantities of foreign DNA, and that this remains consistent throughout several soil types (see Figure S1, Supporting Information). Therefore, the large between-sample variation that was found cannot be attributed simply to artifacts caused by complex template interactions.

\section{DISCUSSION}

Although the number of studies in soil microbial ecology utilizing HTS techniques is steadily increasing, to our knowledge there have been no studies that have examined the patterns of local, interseasonal and intercontinental variability in soil bacterial and eukaryotic microbial communities simultaneously. This study aimed to characterize temporal changes in soil microbial communities to investigate the relative size of temporal variation compared to spatial variation, and whether the spatiotemporal differences vary between broad taxonomic groups, i.e. bacteria, fungi, protists and metazoa. We found a strong link between spatial distance and community dissimilarity, with a decreasing slope with increasing organism size/complexity, while the intercept shows the opposite trend. Furthermore, correlated community dissimilarities were found between different microbial groups in our interseasonal comparison, indicating these groups change in tandem between time points.

\section{Temporal and spatial patterns of microbial communities}

No consistent pattern was found when comparing samples of a single time point with samples between time points, e.g. bacterial communities of Svartberget (subarctic pine forest) sampled at time points months apart were less dissimilar on average than samples taken in the same instance, while bacterial communities of Brasschaat (temperate oceanic pine forest) displayed the opposite pattern (Fig. 1). This lack of a consistent pattern explains why no significant relationship was found between interseasonal changes in environmental parameters and community changes. It seems that between-sample variation at the local scale plays a large role in shaping observed community dissimilarity patterns. Much of this variation can be attributed to random sampling effects, i.e. the limited sampling from a (spatially) heterogeneous population. This then also comes into account in repeated measurements, as it is nearly impossible to resample the exact same community in a natural setting. Therefore, any repeated sampling campaigns will inevitably measure a combination of temporal and random spatial sampling variation. Our results imply that in some cases this spatial variation can be very large, and make detecting temporal patterns difficult. This would appear to be a feature of soil, in contrast to well-mixed aquatic environments (Gilbert et al. 2012; Rösel, Allgaier and Grossart 2012), a fact that should be taken into account when designing studies addressing temporal dynamics in soil communities. 
Table 2. Turnover and difference in variability in Bray-Curtis dissimilarity of each site for each group, with additional information on the amount of taxa during the 'high' and 'low' season, as well as the percentage of shared OTUs between the two time points.

\begin{tabular}{|c|c|c|c|c|c|}
\hline \multicolumn{6}{|l|}{ Bacteria } \\
\hline Site & Turnover & $\begin{array}{c}\text { Difference in } \\
\text { variability }\end{array}$ & $\begin{array}{l}\text { Diversity high } \\
\text { season }\end{array}$ & $\begin{array}{l}\text { Diversity low } \\
\text { season }\end{array}$ & Shared (\%) \\
\hline Cumberland & 0.252 & 0.121 & 1078 & 1475 & 50.13 \\
\hline Barbeau & 0.347 & 0.190 & 1113 & 619 & 42.58 \\
\hline Brasschaat & 0.253 & 0.139 & 639 & 347 & 44.11 \\
\hline Dorinne & 0.184 & 0.081 & 1690 & 1748 & 57.88 \\
\hline Garraf & 0.136 & 0.043 & 1693 & 1810 & 62.53 \\
\hline Reykir & 0.265 & 0.092 & 1415 & 1797 & 52.68 \\
\hline Lonzee & 0.205 & 0.098 & 2715 & 2554 & 61.58 \\
\hline Nouragues_B & 0.104 & 0.016 & 1187 & 1218 & 52.95 \\
\hline Nouragues_T & 0.158 & 0.037 & 994 & 1030 & 46.62 \\
\hline Paracou_B & 0.125 & 0.032 & 1164 & 1018 & 53.37 \\
\hline Paracou_T & 0.154 & 0.036 & 1057 & 1217 & 54.2 \\
\hline Prades & 0.198 & 0.029 & 1857 & 2016 & 57.76 \\
\hline Svartberget & 0.211 & 0.003 & 418 & 500 & 51.76 \\
\hline \multicolumn{6}{|l|}{ Fungi } \\
\hline Cumberland & 0.292 & 0.132 & 121 & 110 & 45.16 \\
\hline Barbeau & 0.492 & 0.385 & 72 & 38 & 27.1 \\
\hline Brasschaat & 0.281 & 0.054 & 68 & 43 & 40.74 \\
\hline Dorinne & 0.327 & 0.035 & 98 & 115 & 46.18 \\
\hline Garraf & 0.331 & 0.090 & 101 & 83 & 52.94 \\
\hline Reykir & 0.292 & 0.198 & 111 & 99 & 47.13 \\
\hline Lonzee & 0.484 & 0.329 & 91 & 111 & 49.35 \\
\hline Nouragues_B & 0.177 & 0.028 & 152 & 159 & 47.63 \\
\hline Nouragues_T & 0.279 & 0.014 & 120 & 100 & 41.77 \\
\hline Paracou_B & 0.294 & 0.080 & 131 & 163 & 45.17 \\
\hline Paracou_T & 0.408 & 0.167 & 109 & 147 & 41.78 \\
\hline Prades & 0.259 & 0.089 & 88 & 98 & 47.2 \\
\hline Svartberget & 0.240 & 0.030 & 64 & 73 & 55.74 \\
\hline \multicolumn{6}{|l|}{ Metazoa } \\
\hline Cumberland & 0.413 & 0.158 & 46 & 48 & 35.71 \\
\hline Barbeau & 0.420 & 0.201 & 58 & 17 & 26.05 \\
\hline Brasschaat & 0.273 & 0.160 & 35 & 25 & 45.12 \\
\hline Dorinne & 0.522 & 0.313 & 14 & 12 & 34.04 \\
\hline Garraf & 0.374 & 0.150 & 29 & 26 & 38.13 \\
\hline Reykir & 0.350 & 0.101 & 53 & 45 & 46.7 \\
\hline Lonzee & 0.485 & 0.279 & 17 & 19 & 24.14 \\
\hline Nouragues_B & 0.440 & 0.071 & 69 & 47 & 28.87 \\
\hline Nouragues_T & 0.427 & 0.072 & 67 & 28 & 21.91 \\
\hline Paracou_B & 0.484 & 0.064 & 57 & 36 & 28.63 \\
\hline Paracou_T & 0.354 & 0.022 & 57 & 33 & 28.31 \\
\hline Prades & 0.333 & 0.133 & 52 & 59 & 51.72 \\
\hline Svartberget & 0.344 & 0.058 & 42 & 58 & 60.5 \\
\hline \multicolumn{6}{|l|}{ Protists } \\
\hline Cumberland & 0.353 & 0.154 & 450 & 481 & 47.73 \\
\hline Barbeau & 0.498 & 0.355 & 308 & 65 & 19.97 \\
\hline Brasschaat & 0.431 & 0.151 & 178 & 105 & 35.68 \\
\hline Dorinne & 0.312 & 0.052 & 365 & 408 & 48.54 \\
\hline Garraf & 0.237 & 0.043 & 328 & 322 & 56.36 \\
\hline Reykir & 0.332 & 0.138 & 392 & 346 & 47.31 \\
\hline Lonzee & 0.326 & 0.203 & 373 & 407 & 45.68 \\
\hline Nouragues_B & 0.237 & 0.001 & 320 & 307 & 41.63 \\
\hline Nouragues_T & 0.361 & 0.041 & 244 & 153 & 36.86 \\
\hline Paracou_B & 0.251 & 0.048 & 298 & 284 & 44.15 \\
\hline Paracou_T & 0.311 & 0.053 & 290 & 246 & 43.9 \\
\hline Prades & 0.219 & 0.051 & 460 & 442 & 53.84 \\
\hline Svartberget & 0.271 & 0.048 & 183 & 198 & 50.29 \\
\hline
\end{tabular}




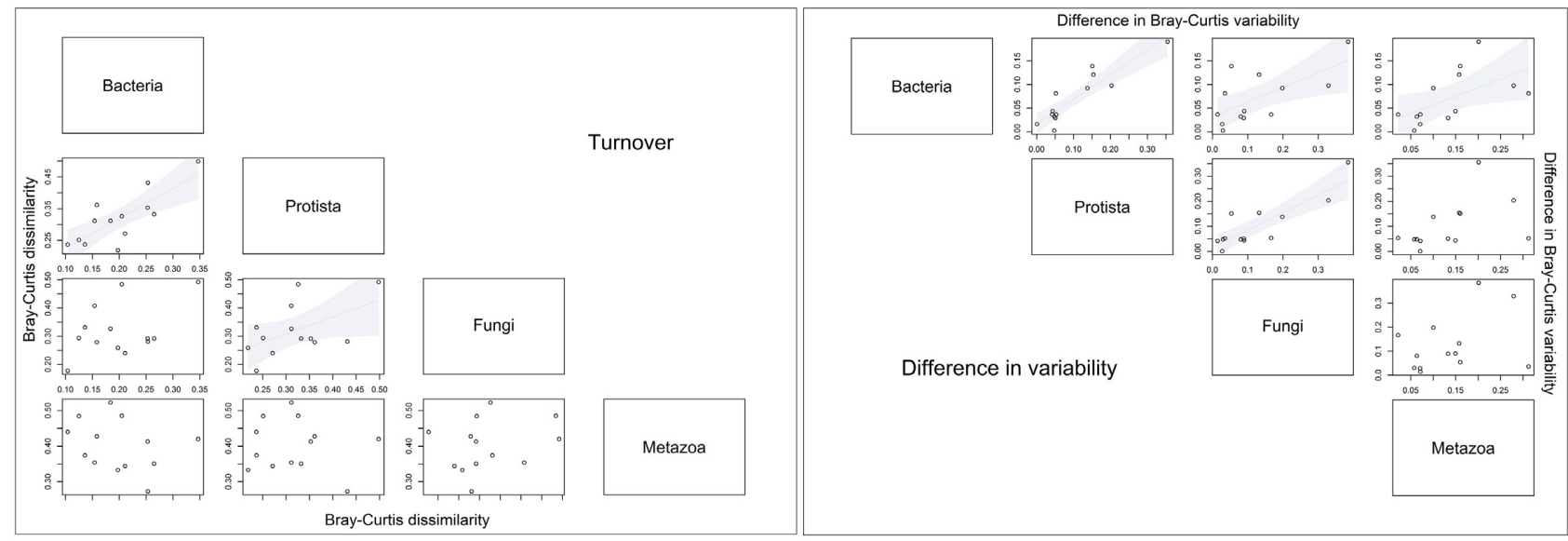

Figure 2. Correlations between microbial groups for turnover (the distance between temporal centroids) on the left and correlation between microbial groups for difference in variability (the difference in spread between time points) on the right. Every dot represents a single site and significant correlations are highlighted by their linear fit and confidence interval (shading)
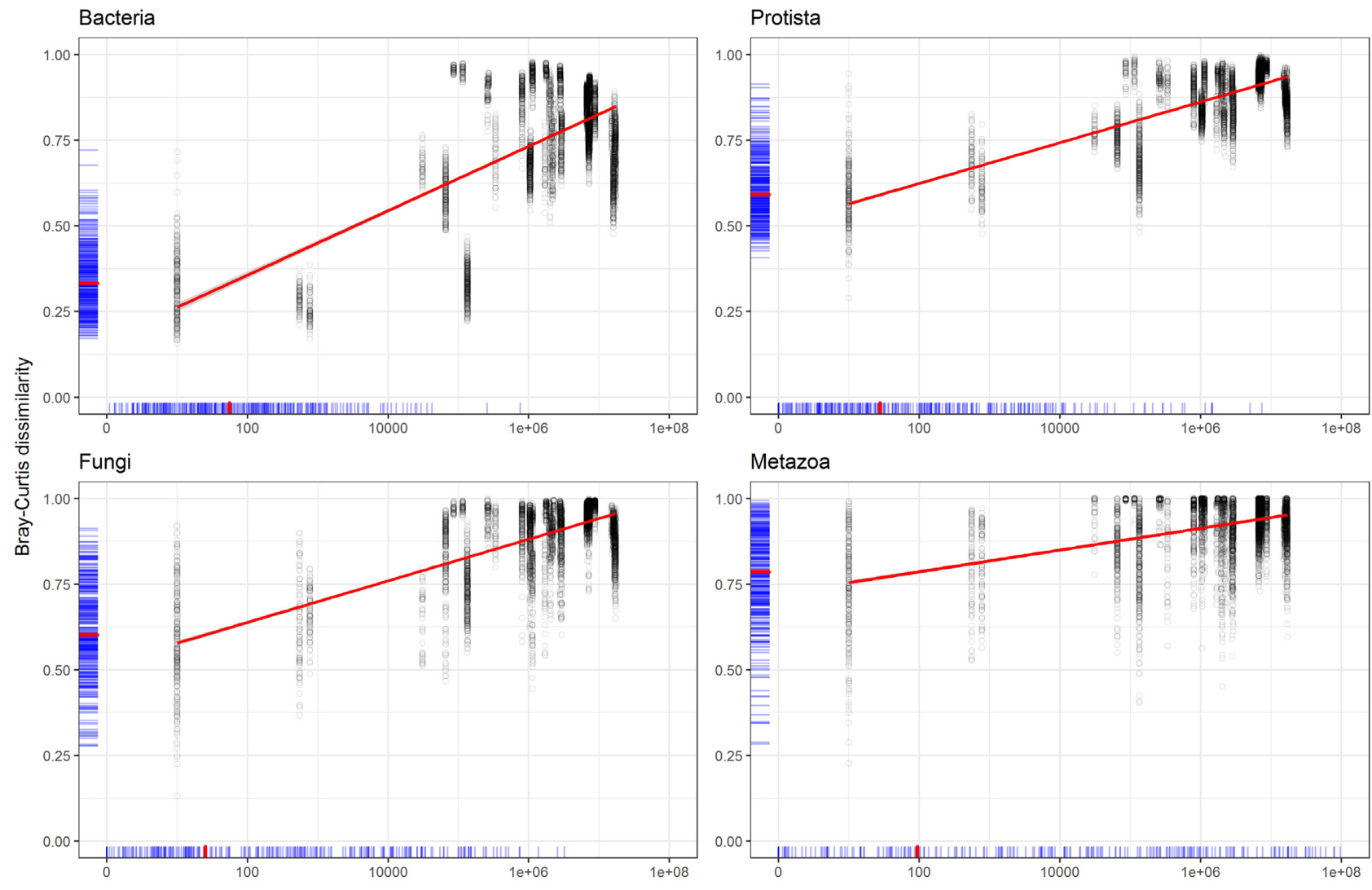

\section{Metazoa}

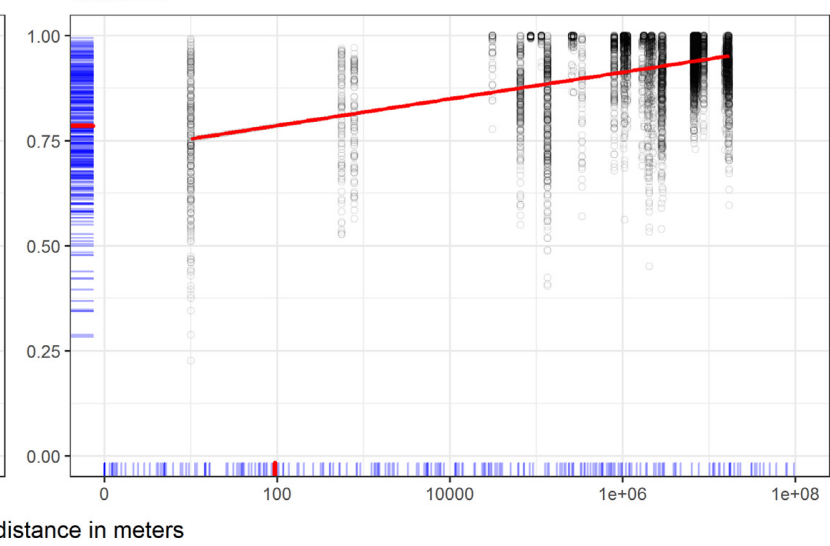

Figure 3. Linear models showing the significant relationship between community dissimilarity and spatial distance. The y-scale shows community dissimilarity while the $x$-axis shows log-transformed spatial distance in meters. The red line shows the model fit and blue rugs on the $y$-axis show all intra-annual within-site community dissimilarities. The rugs on the $x$-axis are projected spatial distances according to the model fit and thus indicate the extent of community variation found in intraannual sampling scaled to that found at sites with increasing distance. Median values for intra-annual community dissimilarity and corresponding calculated spatial distance are highlighted in red. Calculated spatial distance was cut off at 0 as spatial distance cannot go below 0 . Top left: bacteria $\left(R^{2}=0.44\right.$, intercept $=0.17$, slope $=$ $0.094)$, top right: protists $\left(R^{2}=0.57\right.$, intercept $=0.51$, slope $\left.=0.059\right)$, bottom left: fungi $\left(R^{2}=0.49\right.$, intercept $=0.52$, slope $\left.=0.061\right)$ and bottom right: metazoa $\left(R^{2}=0.21\right.$, intercept $=0.72$, slope $=0.032$. 
The lack of a correlation between interseasonal changes in community composition and edaphic parameters contradicts some of the earlier work on temporal changes in soil microbial communities (Bréchet et al. 2018; Buscardo et al. 2018; Lan et al. 2018; Oberholster et al. 2018; Ratcliffe, Bosman and Carnol 2018; Yang et al. 2018; Zhang et al. 2018). These studies found interseasonal changes in community composition or respiration, which were linked to temporal differences in precipitation, amount and quality of litter, and changes in the resident plant community. However, it should be noted that these studies used homogenized composite samples, thereby eliminating some of the local variation introduced by random sampling effects. Given that we only tested for effects of changes in parameters that are distributed relatively equally on the local scale, we cannot rule out that parameters that vary more throughout the year on a small spatial scale such as vegetation (Ramirez et al. 2014; Tedersoo et al. 2016), nutrients and litter fall (Bates et al. 2013; Ramirez et al. 2014; Shen et al. 2014; Shi et al. 2015; Dupont et al. 2016) influenced the microbial community. The amount and quality of litter, for example, change both throughout the year and over small scales $(<1 \mathrm{~m})$. The substrate it provides could be a potential explanatory factor for large local differences in soil microbial communities. The temperate broadleaf site Barbeau displayed some of the largest temporal changes together with some of the largest variation within a time point (autumn), which can be explained by high litter input in autumn. Depending on tree species and site topology, leaf litter can exhibit a very patchy spatial distribution in a given site. Furthermore, the stage of decomposition might also vary on a small local scale.

Despite low evidence of consistent interseasonal turnover and difference in variability of communities, interseasonal changes in both turnover and difference in variability are consistent between some groups but not others. This could indicate a strong linkage between these co-changing groups, or at the very least a parallel response to joint local circumstances or seasons. Large interseasonal changes in protist communities coincided with large changes in bacterial and fungal communities. Many protist species are known to be bacterial consumers, and microbial grazing can exert a strong top-down effect on communities when consumers have a high abundance and growth rate (Lenoir et al. 2007; Crowther, Boddy and Jones 2011). At the same time, bottom-up effects affect grazing communities through the growth rates of the prey microbes. For example, grazed communities might be able to increase their growth rate and maintain high abundances despite predation as long as available nutrients remain high (Couteaux and Bottner 1994; Vedder et al. 1996; Mikola and Setälä 1998). Changing seasons may then affect growth rates of both communities through parameters such as nutrient availability or temperature. No correlation was found between protists and metazoans, possibly as a result of coarse taxonomic resolution that precludes detection of the more subtle and complex relationships that may exist. However, this does not mean that they do not potentially interact with each other. Several protists have been recorded as predators or parasites of metazoans (Adl and Gupta 2006; Jassey et al. 2012; Geisen et al. 2015b; Mitchell 2015), and these interactions might become more obvious when examining data on a more local scale or when looking at more specific groups. Alternatively, primer bias toward protists and fungi, which caused a relative low read number for metazoa, may explain the lack of correlation (see the Supporting Information). We discovered that difference in variability of communities, which can be interpreted as the difference in sample-to-sample variation between time points, has a very strong correlation between bacteria and all other groups, as well as between protists and fungi. As mentioned above, local differences in soil and biotic parameters exert a strong effect on microbial communities (Shi et al. 2015; Dupont et al. 2016; Tedersoo et al. 2016; Bréchet et al. 2018; Buscardo et al. 2018). As communities become more variable on a local scale for one group, it is to be expected that other groups that are interacting with these microbes also become more variable.

In the correlation between community dissimilarity and spatial distance, bacterial communities show the lowest variation at local scales (intercept) and have (perhaps as a consequence) the largest change over spatial distance (slope). The opposite is true for metazoa, with fungi and protists being intermediate. This corresponds with the findings for the temporal scale, with bacteria showing the smallest changes in community composition locally, and metazoa showing the largest. Interestingly, although our distance-decay rates are overall comparable to the ones previously found for microbes, the differences between taxonomic groups contradict some earlier findings, which showed that smaller organisms display the weakest overall trends (Hillebrand et al. 2001; Hillebrand and Azovsky 2001; Zhou et al. 2008; Monroy et al. 2012). For example, Monroy et al. (2012) found that the community dissimilarity-spatial distance relationship was weaker for bacteria when compared to Nematoda, the opposite to our findings comparing bacteria to metazoa. Meyer et al. (2018) recently showed that the main reason for finding weak biogeographic patterns is the sampling extent, i.e. the more thoroughly sampled the community and closer to observing the full community diversity, the steeper the distance-decay rate. This can then also provide an explanation for the results found in our study. Logically, the larger the organism relative to the size of the sample, the more patchy its observed distribution will be. This causes a higher starting dissimilarity between samples, leading to a lower change over spatial distance. This finding is also in line with the results from Zinger et al. (2019), that the amount of variation in community composition explained by environmental variables decreases with increasing propagule size (used as a proxy for organism size) across taxonomic groups. Although they suggest that this is more due to differing dispersal and metabolic rates, we suggest this may also stem from increasing stochasticity when sampling larger organisms, as mentioned above.

\section{CONCLUSION}

To our knowledge, this is the first study to investigate patterns of local, interseasonal and intercontinental variability in soil bacterial and eukaryotic microbial communities simultaneously. We extended and confirmed previous results that organism size greatly affects the distance-decay relationship for community dissimilarity. Regarding temporal variation, we did not detect significant correlations between the changing environment and the associated communities, mostly due to large variation at small spatial scales. These findings demonstrate that temporal and spatial variation work on a similar axis in affecting microbial communities, and that the two cannot be viewed as completely separate.

Our results further show that soil communities displayed correlating community dissimilarities between different microbial groups. Understanding how microbial groups interact with each other and how this influences the response to environmental changes is one of the key questions in microbial ecology. This study provides an important step in solving this question by providing evidence for either a strong linkage between these co-changing groups, or at the very least a parallel response 
to joint local circumstances. This work moves us toward a better understanding of how spatio-temporal variation determines soil microbial community composition and the degree of consistency across different taxonomic groups.

\section{SUPPLEMENTARY DATA}

Supplementary data are available at FEMSEC online.

\section{Data accessibility}

The raw sequences were deposited in the National Centre for Biotechnology Information's (NCBI) Sequence Read Archive (SRA) database under the accesion no. PRJNA605301. Additional information is available from the corresponding author on reasonable request.

\section{ACKNOWLEDGMENTS}

The authors would like to thank Jeff Powell and Catriona Macdonald for providing the DNA extracts and environmental data for the Australian sites. Furthermore, the authors would like to thank Cristina Ariza Carricondo and Lodewijk Lefevre for providing soil samples of the Belgian site Brasschaat, and Claudia Schrauwen for help with the labwork.

\section{AUTHOR CONTRIBUTIONS}

JDG, EV and JTW conceived the idea and designed the analyses. SG provided advice on eukaryotic sequencing. SB, PRFG, LGDLM, BH, IAJ, TM, RO, MOL, JP, BDS and GV established and maintained the field sites; SB, SD, PRFG, LGDLM, NL, TM, RO, JP, BDS and GV conducted the field sampling and/or supplied auxiliary data. JDG performed lab work, bioinformatics and statistical analyses with guidance from EV and JTW. All authors contributed to interpretation of the results, and read and approved the final manuscript

\section{FUNDING}

BDS was supported by the Icelandic Research Fund (ForHotForest; 163272-051) and the Nordic Council of Ministers under the Centre of Advanced Research on Environmental Services from Nordic forest ecosystems (CAR-ES).

The Eucalypt Free Air CO2 Enrichment experiment (EucFACE) was built as an initiative of the Australian Government as part of the Nation-building Economic Stimulus Package, and is supported by the Australian Commonwealth government in collaboration with Western Sydney University.

This work was funded by a fellowship from the Research Foundation Flanders (FWO): FWO-Postdoc fellowship, Phenology underground: seasonality of soil microbial communities and functions (01/10/2013-30/09/2019) (UA nr. 42/FA070400/OZ6423). JDG was funded by a University Research Fund (BOF) from the University of Antwerp: TOP-BOF UA 2014-2017: FORHOT (UA nr. 41/FA070400/FFB140025).

Conflicts of interest. The authors declare that they have no competing interests.

\section{REFERENCES}

Adl MS, Gupta VVSR. Protists in soil ecology and forest nutrient cycling. Can J Forest Res 2006;36:1805-17.
Bahram M, Hildebrand F, Forslund SK et al. Structure and function of the global topsoil microbiome. Nature 2018;560:233-7.

Bates ST, Clemente JC, Flores GE et al. Global biogeography of highly diverse protistan communities in soil. ISME $J$ 2013;7:652-9.

Bernhard JM, Kormas K, Pachiadaki MG et al. Benthic protists and fungi of Mediterranean deep hypsersaline anoxic basin redoxcline sediments. Front Microbiol 2014;5:1-13.

Bittinger K. usedist: Distance Matrix Utilities. R package version 0.1.0. URL: https://CRAN.R-project.org/package=usedist. 2017.

Bréchet LM, Lopez-Sangil L, George C et al. Distinct responses of soil respiration to experimental litter manipulation in temperate woodland and tropical forest. Ecol Evol 2018;8:3787-96.

Buscardo E, Geml J, Schmidt SK et al. Spatio-temporal dynamics of soil bacterial communities as a function of Amazon forest phenology. Sci Rep 2018;8:1-13.

Caporaso JG, Kuczynski J, Stombaugh J et al. QIIME allows analysis of high-throughput community sequencing data. Nat Methods 2010;7:335-6.

Caron DA, Countway PD, Jones AC et al. Marine protistan diversity. Ann Rev Mar Sci 2011;4:467-93.

Chemidlin Prevost-Boure N, Maron PA, Ranjard L et al. Seasonal dynamics of the bacterial community in forest soils under different quantities of leaf litter. Appl Soil Ecol 2011;47: 14-23.

Clarholm M. Protozoan grazing of bacteria in soil-impact and importance. Microb Ecol 1981;7:343-50.

Couteaux MM, Bottner P. Biological Interactions Between Fauna and the Microbial Community in Soils. Chichester: John Wiley \& Sons, 1994.

Crowther TW, Boddy L, Jones TH. Species-specific effects of soil fauna on fungal foraging and decomposition. Oecologia 2011;167:535-45.

Delgado-Baquerizo M, Oliverio AM, Brewer TE et al. A global atlas of the dominant bacteria found in soil. Science 2018a;359: $320-5$.

Delgado-Baquerizo M, Reith F, Dennis PG et al. Ecological drivers of soil microbial diversity and soil biological networks in the Southern Hemisphere. Ecology 2018b;99:583-96.

de Ruiter PC, Neutel A-M, Moore JC. Energetics, patterns of interaction strengths, and stability in real ecosystems. Science 1995;269:1257-60.

Dunthorn M, Kauserud H, Bass D et al. Yeasts dominate soil fungal communities in three lowland Neotropical rainforests. Environ Microbiol Rep 2017;9:668-75.

Dupont AÖC, Griffiths RI, Bell $\mathrm{T}$ et al. Differences in soil micro-eukaryotic communities over soil $\mathrm{pH}$ gradients are strongly driven by parasites and saprotrophs. Environ Microbiol 2016;18:2010-24.

Edgar RC. Search and clustering orders of magnitude faster than BLAST. Bioinformatics 2010;26:2460-1.

Edgar RC. UPARSE: highly accurate OTU sequences from microbial amplicon reads. Nat Methods 2013;10:996.

Ekelund F, Rønn R. Notes on protozoa in agricultural soil with emphasis on heterotrophic flagellates and naked amoebae and their ecology. FEMS Microbiol Rev 1994;15:321-53.

Finlay BJ, Black HIJ, Brown S et al. Estimating the growth potential of the soil protozoan community. Protist 2000;151:69-80.

Foissner W. Protozoa as bioindicators in agroecosystems, with emphasis on farming practices, biocides, and biodiversity. Agric Ecosyst Environ 1997;62:93-103.

Geisen S, Koller R, Hünninghaus $\mathrm{M}$ et al. The soil food web revisited: diverse and widespread mycophagous soil protists. Soil Biol Biochem 2016;94:10-8. 
Geisen S, Laros I, Vizcaíno A et al. Not all are free-living: high-throughput DNA metabarcoding reveals a diverse community of protists parasitizing soil metazoa. Mol Ecol 2015a;24:4556-69.

Geisen S, Mitchell EAD, Wilkinson DM et al. Soil protistology rebooted: 30 fundamental questions to start with. Soil Biol Biochem 2017;111:94-103.

Geisen S, Rosengarten J, Koller R et al. Pack hunting by a common soil amoeba on nematodes. Environ Microbiol 2015b;17: 4538-46.

Gilbert JA, Steele JA, Caporaso JG et al. Defining seasonal marine microbial community dynamics. ISME J 2012;6:298-308.

Guillou L, Bachar D, Audic S et al. The Protist Ribosomal Reference database (PR2): a catalog of unicellular eukaryote small sub-unit rRNA sequences with curated taxonomy. Nucleic Acids Res 2013;41:D597-604.

Hijmans RJ. geosphere: Spherical Trigonometry. R package version 1.57. URL: https://CRAN.R-project.org/package=geosphere. 2017.

Hillebrand H, Azovsky AI. Body size determines the strength of the latitudinal diversity gradient. Ecography 2001;24:251-6.

Hillebrand H, Watermann F, Karez R et al. Differences in species richness patterns between unicellular and multicellular organisms. Oecologia 2001;126:114-24.

Jassey VEJ, Shimano S, Dupuy C et al. Characterizing the feeding habits of the testate amoebae Hyalosphenia papilio and Nebela tincta along a narrow "Fen-Bog" gradient using digestive vacuole content and13C and15N isotopic analyses. Protist 2012;163:451-64.

Kennedy K, Hall MW, Lynch MDJ et al. Evaluating bias of Illumina-based bacterial 16S rRNA gene profiles. Appl Environ Microb 2014;80:5717-22.

Klindworth A, Pruesse E, Schweer T et al. Evaluation of general 16S ribosomal RNA gene PCR primers for classical and nextgeneration sequencing-based diversity studies. Nucleic Acids Res 2013;41:1-11.

Lan G, Li Y, Lesueurd D et al. Seasonal changes impact soil bacterial communities in a rubber plantation on Hainan Island, China. Sci Total Environ 2018;626:826-34.

Lauber CL, Hamady M, Knight R et al. Pyrosequencing-based assessment of soil pH as a predictor of soil bacterial community structure at the continental scale. Appl Environ Microb 2009;75:5111-20.

Lauber CL, Ramirez KS, Aanderud Z et al. Temporal variability in soil microbial communities across land-use types. ISME J 2013;7:1641-50.

Lazzaro A, Hilfiker D, Zeyer J. Structures of microbial communities in alpine soils: seasonal and elevational effects. Front Microbiol 2015;6:1-13.

Leff JW, Jones SE, Prober SM et al. Consistent responses of soil microbial communities to elevated nutrient inputs in grasslands across the globe. Proc Natl Acad Sci 2015;112: 10967-72.

Lenoir L, Persson T, Bengtsson J et al. Bottom-up or top-down control in forest soil microcosms? Effects of soil fauna on fungal biomass and $\mathrm{C} / \mathrm{N}$ mineralisation. Biol Fertil Soils 2007:43:281-94.

Lie AAY, Liu Z, Hu SK et al. Investigating microbial eukaryotic diversity from a global census: insights from a comparison of pyrotag and full-length sequences of 18S rRNA genes. Appl Environ Microb 2014;80:4363-73.

Logares R, Haverkamp THA, Kumar S et al. Environmental microbiology through the lens of high-throughput DNA sequencing: synopsis of current platforms and bioinformatics approaches. J Microbiol Methods 2012;91: 106-13.

López-Escardó D, Paps J, De Vargas C et al. Metabarcoding analysis on European coastal samples reveals new molecular metazoan diversity. Sci Rep 2018;8:1-14.

Maestre FT, Delgado-Baquerizo M, Jeffries TC et al. Increasing aridity reduces soil microbial diversity and abundance in global drylands. Proc Natl Acad Sci 2015;112:201516684.

Mahé F, Mayor J, Bunge J et al. Comparing high-throughput platforms for sequencing the V4 region of SSU-rDNA in environmental microbial eukaryotic diversity surveys. J Eukaryot Microbiol 2015;62:338-45.

Mahé F, De Vargas C, Bass D et al. Parasites dominate hyperdiverse soil protist communities in Neotropical rainforests. Nat Ecol Evol 2017;1:1-8.

Meyer KM, Memiaghe H, Korte L et al. Why do microbes exhibit weak biogeographic patterns? ISME J 2018;12:1404-13.

Mikola J, Setälä H. No evidence of trophic cascades in an experimental microbial-based soil food web. Ecology 1998;79: 153-64.

Mitchell EAD. Pack hunting by minute soil testate amoebae: nematode hell is a naturalist's paradise. Environ Microbiol 2015;17:4145-7.

Monroy F, van der Putten WH, Yergeau E et al. Community patterns of soil bacteria and nematodes in relation to geographic distance. Soil Biol Biochem 2012;45:1-7.

Oberholster T, Vikram S, Cowan D et al. Key microbial taxa in the rhizosphere of sorghum and sunflower grown in crop rotation. Sci Total Environ 2018;624:530-9.

Oksanen J, Blanchet FG, Friendly M et al. vegan: Community Ecology Package. R package version 2.5-5. URL: https://CRAN.R-project.o rg/package=vegan 2019.

Pawlowski J, Esling P, Lejzerowicz F et al. Environmental monitoring through protist next-generation sequencing metabarcoding: assessing the impact of fish farming on benthic foraminifera communities. Mol Ecol Resour 2014;14: 1129-40.

Prober SM, Leff JW, Bates ST et al. Plant diversity predicts beta but not alpha diversity of soil microbes across grasslands worldwide. Ecol Lett 2015;18:85-95.

Quast C, Pruesse E, Yilmaz P et al. The SILVA ribosomal RNA gene database project: improved data processing and web-based tools. Nucleic Acids Res 2013;41:D590-6.

R Core Team. R: A Language and Environment for Statistical Computing. R Foundation for Statistical Computing, Vienna, Austria. URL: https://www.R-project.org/ 2019.

Ramirez KS, Leff JW, Barberán A et al. Biogeographic patterns in below-ground diversity in New York City's Central Park are similar to those observed globally. Proc Biol Sci 2014;281:20141988.

Ratcliffe S, Bosman B, Carnol M. Spatial and temporal variability of biological indicators of soil quality in two forest catchments in Belgium. Appl Soil Ecol 2018;126: 148-59.

Rognes T, Flouri T, Nichols B et al. VSEARCH: a versatile open source tool for metagenomics. PeerJ 2016;4:e2584.

Rösel S, Allgaier M, Grossart HP. Long-term characterization of free-living and particle-associated bacterial communities in lake tiefwaren reveals distinct seasonal patterns. Microb Ecol 2012;64:571-83.

Rousk J, Bååth E, Brookes PC et al. Soil bacterial and fungal communities across a $\mathrm{pH}$ gradient in an arable soil. ISME J 2010;4:1340. 
Shen C, Liang W, Shi Y et al. Contrasting elevational diversity patterns between eukaryotic soil microbes and plants. Ecology 2014;95:3190-202.

Shi Y, Xiang X, Shen C et al. Vegetation-associated impacts on Arctic tundra bacterial and microeukaryotic communities. Appl Environ Microb 2015;81:492-501.

Stoeck T, Bass D, Nebel M et al. Multiple marker parallel tag environmental DNA sequencing reveals a highly complex eukaryotic community in marine anoxic water. Mol Ecol 2010;19:21-31.

Talbot JM, Bruns TD, Taylor JW et al. Endemism and functional convergence across the North American soil mycobiome. Proc Natl Acad Sci 2014;111:6341-6.

Tedersoo L, Bahram M, Cajthaml $\mathrm{T}$ et al. Tree diversity and species identity effects on soil fungi, protists and animals are context dependent. ISME J 2016;10:346-62.

Tedersoo L, Bahram M, Põlme S et al. Global diversity and geography of soil fungi. Science 2014;346:1256688.

Treseder KK, Maltz MR, Hawkins BA et al. Evolutionary histories of soil fungi are reflected in their large-scale biogeography. Ecol Lett 2014;17:1086-93.

Vedder B, Kampichler C, Bachmann G et al. Impact of faunal complexity on microbial biomass and $\mathrm{N}$ turnover in field mesocosms from a spruce forest soil. Biol Fertil Soils 1996;22: 22-30.

Yang F, Wu J, Zhang D et al. Soil bacterial community composition and diversity in relation to edaphic properties and plant traits in grasslands of southern China. Appl Soil Ecol 2018;128:43-53.

Yao H, Bowman D, Shi W. Seasonal variations of soil microbial biomass and activity in warm- and cool-season turfgrass systems. Soil Biol Biochem 2011;43:1536-43.

Zhang J, Li M, Jia $\mathrm{K}$ et al. Seasonal variation rather than stand age determines bacterial diversity in the rhizosphere of wolfberry (Lycium barbarum L.) associated with soil degradation. J Soils Sediments 2018;18: 1518-29.

Zhou J, Deng Y, Shen L et al. Temperature mediates continentalscale diversity of microbes in forest soils. Nat Commun 2016;7:1-10.

Zhou J, Kang S, Schadt CW et al. Spatial scaling of functional gene diversity across various microbial taxa. Proc Natl Acad Sci 2008;105:7768-73.

Zinger L, Taberlet $\mathrm{P}$, Schimann $\mathrm{H}$ et al. Body size determines soil community assembly in a tropical forest. Mol Ecol 2019;28:528-43. 